\title{
Aerobic exercise interventions reduce blood pressure in patients after stroke or transient ischaemic attack: a systematic review and meta-analysis
}

\author{
Cheng Wang, ${ }_{1}^{1}$ Jessica Redgrave, ${ }_{1}^{2}$ Mohsen Shafizadeh, ${ }^{3}$ Arshad Majid, ${ }_{1}^{1,4}$ Karen Kilner, ${ }^{5}$ \\ Ali N Ali ${ }^{1,6}$
}

\begin{abstract}
- Additional material is published online only. To view, please visit the journal online (http://dx.doi.org/10.1136/ bjsports-2017-098903).

${ }^{1}$ Faculty of Medicine Dentistry and Health, University of Sheffield, Sheffield, UK ${ }^{2}$ Department of Neurosciences, Sheffield Teaching Hospitals NHS Foundation Trust, Sheffield, UK

${ }^{3}$ Sheffield Hallam University, Academy of Sport and Physical Activity, Sheffield, UK ${ }^{4}$ Sheffield NIHR Biomedical Research Centre, Sheffield, UK ${ }^{5}$ Sheffield Hallam University, Centre for Health and Social Care Research, Sheffield, UK 'Sheffield Teaching Hospitals NHS Foundation Trust, Geriatrics and Stroke Medicine, Sheffield, UK
\end{abstract}

\section{Correspondence to}

Dr Ali N Ali, Department of Geriatrics and Stroke Medicine, Sheffield Teaching Hospitals NHS Foundation Trust, Sheffield S10 2JF, UK; ali.ali@sth.nhs.uk

Accepted 10 April 2018 Published Online First 9 May 2018

Check for updates

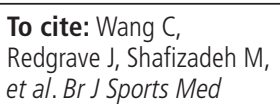

\section{ABSTRACT}

Objective Secondary vascular risk reduction is critical to preventing recurrent stroke. We aimed to evaluate the effect of exercise interventions on vascular risk factors and recurrent ischaemic events after stroke or transient ischaemic attack (TIA).

Design Intervention systematic review and metaanalysis.

Data sources OVID MEDLINE, PubMed, The Cochrane Library, Web of Science, The National Institute for Health and Care Excellence, TRIP Database, CINAHL, PsycINFO, SCOPUS, UK Clinical Trials Gateway and the China National Knowledge Infrastructure were searched from 1966 to October 2017

Eligibility criteria Randomised controlled trials evaluating aerobic or resistance exercise interventions on vascular risk factors and recurrent ischaemic events among patients with stroke or TIA, compared with control.

Results Twenty studies ( $n=1031)$ were included. Exercise interventions resulted in significant reductions in systolic blood pressure (SBP) $-4.30 \mathrm{~mm} \mathrm{Hg}(95 \% \mathrm{Cl}$ -6.77 to -1.83$)$ and diastolic blood pressure $-2.58 \mathrm{~mm}$ $\mathrm{Hg}(95 \% \mathrm{Cl}-4.7$ to -0.46$)$ compared with control. Reduction in SBP was most pronounced among studies initiating exercise within 6 months of stroke or TIA $(-8.46 \mathrm{~mm} \mathrm{Hg}, 95 \% \mathrm{Cl}-12.18$ to -4.75 vs $-2.33 \mathrm{~mm}$ $\mathrm{Hg}, 95 \% \mathrm{Cl}-3.94$ to -0.72$)$, and in those incorporating an educational component $(-7.81 \mathrm{~mm} \mathrm{Hg}, 95 \% \mathrm{Cl}$ -14.34 to -1.28 vs $-2.78 \mathrm{~mm} \mathrm{Hg}, 95 \% \mathrm{Cl}-4.33$ to $-1.23)$. Exercise was also associated with reductions in total cholesterol $(-0.27 \mathrm{mmol} / \mathrm{L}, 95 \% \mathrm{Cl}-0.54$ to 0.00$)$, but not fasting glucose or body mass index. One trial reported reductions in secondary vascular events with exercise, but was insufficiently powered.

Summary Exercise interventions can result in clinically meaningful blood pressure reductions, particularly if initiated early and alongside education.

\section{INTRODUCTION}

Stroke is the second leading cause of death and adult disability worldwide, affecting approximately 15 million individuals annually. ${ }^{1}$ One in five people who suffer a stroke die within the first 30 days ${ }^{2}$, and over $40 \%$ of the remainder are left functionally dependent at 6 months. ${ }^{3}$ Annual direct and indirect costs to the UK are estimated at $£ 9$ billion ${ }^{4}$, with costs of $€ 38$ billion across Europe. ${ }^{5}$ These patients typically have atherosclerotic disease and classical vascular risk factors such as hypertension, diabetes, dyslipidemia, obesity and physical inactivity; that are also seen in patients with coronary heart disease (CHD). One in four strokes are recurrent, ${ }^{6}$ associated with greater morbidity, mortality and economic consequences $^{7}$ than primary stroke. Therefore, secondary stroke prevention is paramount.

Evidence relating to the secondary prevention of stroke has evolved concentrating on pharmacological (antithrombotics, ${ }^{8}$ antihypertensives, ${ }^{9}$ lipid-lowering ${ }^{10}$ and surgical (carotid endarterectomy, stenting) ${ }^{11}$ interventions. The evidence base for non-pharmacological interventions, particularly exercise, is less clear. Physical inactivity is an independent predictor of primary stroke. ${ }^{12}$ Despite this, only around a half of adults over the age of 65 years in the UK meet nationally recommended levels of weekly physical activity $(150 \mathrm{~min}$ of moderate intensity activity, eg, brisk walking), ${ }^{13}$ and this declines further after stroke or transient ischaemic attack (TIA). ${ }^{14}$ There is overwhelming evidence that aerobic exercise and health education delivered as cardiac rehabilitation, reduces blood pressure, re-infarction rates, cardiovascular and overall mortality among patients with CHD. ${ }^{15}$ Cardiac rehabilitation is now an established part of CHD management, with the US and UK registry data showing sustained improvements in physical activity 1 year after cardiac rehabilitation. ${ }^{16} 17$ Due to the similarities between coronary and cerebrovascular disease, risk factor management after stroke or TIA could benefit from aligning to a similar structure to that of CHD.

Physical fitness training after stroke is safe and improves walking speed and balance. ${ }^{18}$ However, less attention has been paid to whether exercise can reduce secondary vascular risk. A Cochrane review conducted in $2013^{19}$ and systematic review in $2014^{20}$ only included two randomised controlled trials (RCTs), the results of which were inconclusive regarding the impact of exercise on secondary vascular risk. Since then, interest in this area has increased. This systematic review aims to evaluate the effect of exercise interventions on secondary vascular risk factors (blood pressure, lipid profile, plasma fasting glucose and body mass index (BMI)) and risk of recurrent stroke.

\section{METHODS}

This study was undertaken in accordance with the Preferred Reporting Items for Systematic Reviews and Meta-Analyses guideline. ${ }^{21}$ Operational definitions were detailed prior to search initiation based 
on recommendations from the Cochrane Handbook of Systematic Reviews on Interventions. ${ }^{22}$

\section{Selection criteria}

We included studies evaluating adults (aged $>18$ years) who had suffered a stroke (ischaemic or haemorrhagic) or TIA according to the WHO definition. ${ }^{23}$ Only RCTs assessing the effects of exercise or physical fitness interventions compared with usual care or sham were included. Exercise therapy included cardiorespiratory (aimed at improving fitness), resistance (aimed at improving muscle strength or endurance) or mixed components. Interventions termed 'aerobic' involved a clear aim to enhance physical fitness by stimulating heart rate and respiratory rate, for example, running, walking, circuits, cycling. Studies of physical therapy (therapies used to promote, maintain or restore physical function, eg, walking, balance, etc), were only included if they involved cardiorespiratory or resistance components. Only studies reporting on systolic blood pressure (SBP), diastolic blood pressure (DBP), total cholesterol (TC), low-density lipoprotein cholesterol (LDL-C), high-density lipoprotein cholesterol (HDLC), fasting glucose (FG), BMI or secondary cerebrovascular events and cardiovascular death were included.

\section{Search strategy}

Electronic databases search included OVID MEDLINE, PubMed, Web of Science, The Cochrane Library, The National Institute for Health and Care Excellence, TRIP Database, CINAHL, PsycINFO, SCOPUS, UK Clinical Trials Gateway and the China National Knowledge Infrastructure, from 1966 to October 2017. Selected medical subject headings were combined with free text terms relating to stroke (eg, cerebrovascular accident, cerebral infarction), TIA (eg, transient ischaemic attack, ministroke), exercise (eg, physical activity, aerobic exercise) and secondary prevention (eg, outcomes, vascular events, death, BP, lipid profiles, glucose, weight or BMI), to create a search strategy finalised for MEDLINE (see online supplementary material). This was adjusted for use in other databases using appropriate Boolean operators and search symbols. Chinese translations were used for Chinese databases (see online supplementary material). Reference lists of all relevant studies and systematic reviews were scanned for additional potentially relevant studies. Our search did not extend to grey literature.

\section{Study selection and data extraction}

Two review authors (CW and ANA) independently eliminated duplicate and irrelevant studies from title and abstract. Full texts of the remaining studies were obtained and critically reviewed by both authors independently for inclusion. Both reviewers independently extracted data into a prepiloted spreadsheet. Data included participant demographics, study setting and timeframe, intervention arms and their descriptors and outcome measures. The Consensus on Exercise Reporting Template (CERT) ${ }^{24}$ was used to characterise the reporting of exercise specifics (eg, equipment required, group vs individual, level of supervision, etc) (see online supplementary material). Data required for meta-analysis were collected, including mean differences and associated SD between baseline and postintervention outcome assessments. Basic unit conversions for lipid and glycaemic profiles were undertaken to ensure international system of units for analysis (see online supplementary material). Discrepancies in study selection or data extraction were discussed by both reviewers and adjudicated by a third reviewer (MS).

\section{Quality appraisal}

All studies meeting inclusion criteria for the review were assessed independently by both reviewers (CW and ANA) for risk of bias using a checklist developed by the Cochrane Collaboration establishing bias attributable to patient selection, randomisation, blinding, attrition and reporting. ${ }^{25}$ Studies at high risk of bias in one or more domains were classed as high risk overall, while those with unclear information in any domain were classed as moderate risk. Low-risk studies met low-risk criteria for each domain. Quality assessment of evidence for each outcome measure that pooled data (SBP, DBP, TC, LDL-C, HDL-C, FG and $\mathrm{BMI}$ ) was also undertaken using the GRADE (Grading of Recommendations, Assessment, Development and Evaluations) approach. ${ }^{26}$ An initial grading of 'high quality' for RCTs was downgraded by one level for serious concerns (or by two levels for very serious concerns) about the risk of bias, inconsistency, indirectness, imprecision or publication bias relating to combined evidence for each outcome (see online supplementary material).

\section{Data analysis}

A qualitative synthesis of study characteristics was undertaken. Statistical analyses of outcome data were performed using RevMan 5 (V.5.3 from the Cochrane Collaboration). Meta-analyses for continuous variables (SBP, DBP, TC, LDL-C, HDL-C, FG and $\mathrm{BMI}$ ) used the mean difference and the SD of the mean difference, between intervention and control groups. Not all trials reported changes in the SDs preintervention and postintervention. In these instances, trial authors were contacted directly to request missing data, and where this was not possible, we used indirect methods to estimate missing values. ${ }^{27}$ We calculated the $\mathrm{I}^{2}$ statistic to assess statistical heterogeneity. ${ }^{28}$ A random effects model was applied due to anticipated heterogeneity in population (stroke severity, TIA) and exercise interventions. For meta-analysis of outcomes reported by 10 trials or more, we assessed publication bias using tests by Egger $e t a l^{29}$ and Begg and Mazumdar ${ }^{30}$ in STATA statistical software (V.14.0, StataCorp), although we acknowledge that funnel plot asymmetry can be due to reasons other than publication bias. Prespecified subgroup analyses were undertaken to estimate the effects of variables such as inclusion of studies at high risk of bias, the effect of early (within 6 months) versus late (after 6 months) exercise initiation, incorporation of health education and participant type (TIA/non-disabling stroke vs severe stroke). Further details on the complete statistical tests used are available in the online supplementary material.

\section{RESULTS}

Following removal of duplicates, 11899 citations were identified; from which 39 full-ext articles were retrieved (figure 1). After exclusions were applied, 20 RCTs were retained for review and meta-analysis involving 1031 patients. ${ }^{31-50}$

\section{Quality appraisal}

Ten studies were at low risk of bias, ${ }^{33} 34373943-464850$ seven were at moderate risk of bias ${ }^{31}$ and three were at high risk of bias ${ }^{35} 3842$ (table 1). GRADE assessment of the quality of evidence for each meta-analysed outcome ranged from moderate to very low (see online supplementary material).

\section{Study characteristics}

Twenty selected RCTs recruited 1031 patients from 10 countries, details of which are summarised in table 2. Average ages of study participants ranged from 52 to 69 years. Sixteen trials involved only patients who had suffered 


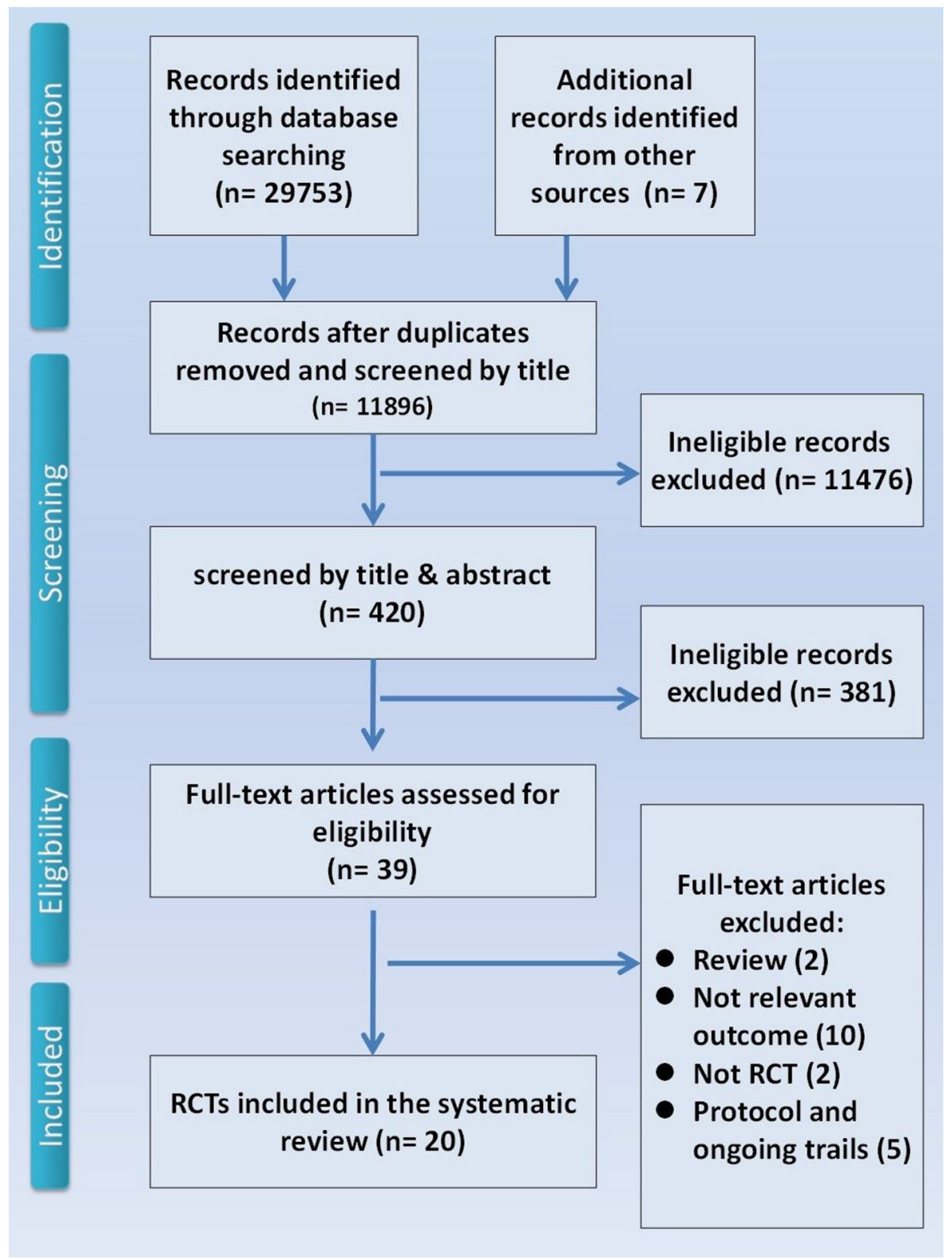

Figure 1 Flow diagram for study selection process. RCT, randomised controlled trial.

stroke, ${ }^{31-3638-404244-4648-50}$ two included only patients with TIA ${ }^{3747}$ and two included both stroke and TIA. ${ }^{41} 43$ Eleven trials recruited

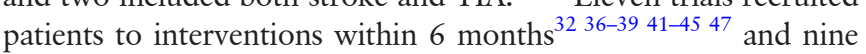
trials after 6 months $s^{3133-35404648-50}$ of the index event. The majority of studies recruited only ambulant participants (with or without walking aids), while five studies recruited patients with more severe stroke. ${ }^{31-334445}$ Five studies excluded patients on the basis of cognitive impairment. ${ }^{35} 38404348$ Exercise interventions were compared with usual care in 12 studies, ${ }^{32} 3436-38$ 40-42 47-49 therapeutic activities in 4 studies (eg, standing and balance training), ${ }^{3544-46}$ stretching in 3 studies $^{313350}$ and an education programme in 1 study. ${ }^{39}$ Exercise interventions varied considerably as reported according to the CERT (see online supplementary material). Overall programme duration ranged from 6 weeks to 6 months and weekly frequency of sessions from 1 to 5 , with each session ranging in duration from 30 to $90 \mathrm{~min}$. Most exercise interventions involved $3 \times 1$ hourly sessions per week (mean weekly minutes $=175$, median $=180$ ), but minutes of weekly programmed exercise activity ranged from 60 to $350 \mathrm{~min}$. Eleven studies involved graded increases in exercise duration or intensity $31323537394046-50$; only three did not include target heart rate changes. ${ }^{394149}$ Four interventions included health educational, 34373843 and only two involved any home programme content. ${ }^{37} 39$ Studies reported poorly on qualifications of supervising health professionals, intervention fidelity and whether exercise was delivered individually or in groups. All studies reported outcome measures relevant to secondary vascular risk, most commonly blood pressure (13 studies), with lipid profile, fasting glucose and BMI reported in at least eight studies. One study reported on vascular events rates. ${ }^{37}$ Two studies referred to the same intervention and group of patients, but reported on different outcome measures, ${ }^{44} 45$ thus were viewed as one study for statistical purposes.

\section{QUANTITATIVE SYNTHESIS (META-ANALYSIS)}

Blood pressure

Data on SBP and DBP were pooled from 12 studies including 606 patients (intervention $\mathrm{n}=305$ )..$^{313234-37394043474850}$ Blood pressure data from one study ${ }^{40}$ did not include measures of 
Table 1 Risk of bias assessed using Cochrane risk of bias tool (high, low, unclear)

\begin{tabular}{|c|c|c|c|c|c|c|c|c|}
\hline Study & $\begin{array}{l}\text { Sequence } \\
\text { generation }\end{array}$ & $\begin{array}{l}\text { Allocation } \\
\text { concealment }\end{array}$ & $\begin{array}{l}\text { Blinding of } \\
\text { participants }\end{array}$ & $\begin{array}{l}\text { Blinding of } \\
\text { outcome } \\
\text { assessment }\end{array}$ & $\begin{array}{l}\text { Incomplete } \\
\text { outcome data } \\
\text { addressed }\end{array}$ & $\begin{array}{l}\text { Free of selective } \\
\text { outcome reporting }\end{array}$ & Other bias & Risk of bias \\
\hline Potempa et al ${ }^{31}$ & Unclear & Unclear & Unclear & Unclear & Low & Low & Low & Moderate \\
\hline Katz-Leurer et $a l^{32}$ & Low & Unclear & Low & Unclear & Low & Low & Low & Moderate \\
\hline Ivey et $\left.a\right|^{33}$ & Low & Low & Low & Low & Low & Low & Low & Low \\
\hline Lennon et $a l^{34}$ & Low & Low & Low & Low & Low & Low & Low & Low \\
\hline Rimmer et $a l^{35}$ & High & Unclear & Low & Unclear & Low & Low & Low & High \\
\hline Toledano-Zarhi et a/ ${ }^{36}$ & Unclear & Unclear & Unclear & Unclear & Low & Low & Low & Moderate \\
\hline Faulkner et $a l^{37}$ & Low & Low & Low & Low & Low & Low & Low & Low \\
\hline Liu and $\operatorname{Yan}^{38}$ & High & Unclear & Low & Low & Unclear & Unclear & Unclear & High \\
\hline Kono et a ${ }^{39}$ & Low & Low & Low & Low & Low & Low & Low & Low \\
\hline Jin et $a l^{40}$ & Low & Low & Low & Unclear & Unclear & Low & Low & Moderate \\
\hline Boss et al ${ }^{41}$ & Unclear & Unclear & Yes & Yes & Low & Low & Low & Moderate \\
\hline Xinzhou $^{42}$ & High & Unclear & Low & Low & Unclear & Unclear & Unclear & High \\
\hline Kirk et al ${ }^{43}$ & Low & Low & Low & Low & Low & Low & Low & Low \\
\hline Wang et $a l^{44}$ & Low & Low & Low & Low & Low & Low & Low & Low \\
\hline Wang et al ${ }^{44}$ & Low & Low & Low & Low & Low & Low & Low & Low \\
\hline Tang et al ${ }^{46}$ & Low & Low & Low & Low & Low & Low & Low & Low \\
\hline Woolley et al ${ }^{47}$ & Unclear & Low & Low & Low & Unclear & Low & Low & Moderate \\
\hline Lee et $a l^{48}$ & Low & Low & Low & Low & Low & Low & Low & Low \\
\hline Zou et al ${ }^{49}$ & Low & Low & Low & Low & Unclear & Low & Low & Moderate \\
\hline Moore et $a l^{50}$ & Low & Low & Low & Low & Low & Low & Low & Low \\
\hline
\end{tabular}

variability and was excluded from meta-analysis. There were significantly greater reductions in SBP with exercise interventions compared with controls $(-4.30 \mathrm{~mm} \mathrm{Hg}, 95 \% \mathrm{CI}-6.77$ to $-1.83 \mathrm{~mm} \mathrm{Hg}, \mathrm{I}^{2}=33 \%$; GRADE of evidence: moderate level) (figure 2). Both tests by Begg and Mazumdar $(\operatorname{Pr}>|z|=0.373)$ and Egger et al $(\mathrm{P}>|\mathrm{t}|=0.226)$ suggested low risk for publication bias.

There were significantly greater reductions in DBP with exercise interventions compared with controls $(-3.12 \mathrm{~mm} \mathrm{Hg}$, $95 \% \mathrm{CI}-4.89$ to $-1.34 \mathrm{~mm} \mathrm{Hg}$; GRADE of evidence: low level) (figure 3). There was significant heterogeneity $\left(\mathrm{I}^{2}=68 \%\right)$ and evidence of publication bias (test by Begg and Mazumdar $\operatorname{Pr}>|\mathrm{z}|=0.047$, test by Egger et al $\mathrm{P}>|\mathrm{t}|=0.033$, 95\%CI -8.87 to -0.47$)$.

Exercise interventions initiated within 6 months 323637394347 of index stroke or TIA appeared to have a larger effect on SBP $\left(-8.46 \mathrm{~mm} \mathrm{Hg}, 95 \% \mathrm{CI}-12.18\right.$ to $\left.-4.75, \mathrm{I}^{2}=0 \%\right)$ than those initiated after 6 months $(-2.33 \mathrm{~mm} \mathrm{Hg}, 95 \% \mathrm{CI}-3.94$ to $\left.-0.72, \mathrm{I}^{2}=0 \%\right){ }^{31} 3435404850$ The benefits for DBP became nonsignificant with exercise initiated after 6 months $(-2.08 \mathrm{~mm} \mathrm{Hg}$, $95 \% \mathrm{CI}-4.82$ to $0.66, \mathrm{I}^{2}=70 \%$ ). Exercise interventions involving an education component ${ }^{34373943}$ also appeared to have greater effects on SBP $(-7.81 \mathrm{~mm} \mathrm{Hg}, 95 \% \mathrm{CI}-14.34$ to -1.28 , $\left.\mathrm{I}^{2}=40 \%\right)$ compared with those with no education component $\left(-2.78 \mathrm{~mm} \mathrm{Hg}, 95 \% \mathrm{CI}-4.33\right.$ to $\left.-1.23, \mathrm{I}^{2}=0 \%\right)$. $^{31323536474850}$ Exclusion of one study at high risk of bias ${ }^{3535}$ did not significantly effect overall results (see online supplementary material).

\section{Lipid profiles}

Nine RCTs reported on total cholesterol $(n=370$ patients; intervention $n=185$ ) $33-35424345464950$ (figure 4A). Pooled analysis showed significant overall reductions in total cholesterol with exercise compared with control $(-0.27 \mathrm{mmol} / \mathrm{L}, 95 \% \mathrm{CI}-0.54$ to $0.00 \mathrm{mmol} / \mathrm{L}, \mathrm{I}^{2}=70 \%, \mathrm{p}=0.05$; GRADE of evidence: low). This beneficial effect however disappeared with the exclusion of two studies that were at high risk of bias ${ }^{35} 42(-0.13 \mathrm{mmol} / \mathrm{L}$, $95 \% \mathrm{CI}-0.40$ to 0.14 , online supplementary material).

Levels of LDL-C have stronger correlations with risk of future stroke and are the main target of lipid-lowering interventions. ${ }^{51}$ Seven RCTs reported on LDL-C ( $\mathrm{n}=303$ patients; intervention $\mathrm{n}=151)^{35394244464950}$ (figure 4B). There was no effect of exercise compared with control $(-0.28 \mathrm{mmol} / \mathrm{L}, 95 \% \mathrm{CI}-0.63$ to $0.07 \mathrm{mmol} / \mathrm{L}, \mathrm{I}^{2}=85 \%$; GRADE of evidence: very low), except among interventions involving health education.

Higher levels of HDL-C have been associated with reduced risk of stroke among diverse populations. ${ }^{52}$ Nine RCTs reported on HDL-C $(n=394$ patients; intervention $n=197$ ) 333539424345464950 (figure 4C). There was no effect of exercise compared with control $(+0.08 \mathrm{mmol} / \mathrm{L}, 95 \% \mathrm{CI}-0.02$ to $0.17 \mathrm{mmol} / \mathrm{L}, \mathrm{I}^{2}=77 \%$; GRADE of evidence: very low).

\section{Fasting blood glucose}

Seven RCTs measured fasting glucose changes ( $n=364$ patients; intervention $n=135) .33373843444649$ There was no effect of exercise on fasting glucose compared with control $(-0.14 \mathrm{mmol} / \mathrm{L}$, $95 \% \mathrm{CI}-0.29$ to 0.01 , moderate heterogeneity $\mathrm{I}^{2}=42 \%$; GRADE of evidence: moderate; figure 4D). Subgroup analyses did not alter these findings.

\section{Body mass index}

EIght RCTs ( $n=446$ patients; intervention $n=225$ ) measured BMI at baseline and follow-up. 34353739434950 There was no effect of exercise on BMI compared with control $\left(-0.0 \mathrm{~kg} / \mathrm{m}^{2}\right.$, $95 \%$ CI -0.26 to $0.25 \mathrm{~kg} / \mathrm{m}^{2}, \mathrm{I}^{2}=32 \%$; GRADE of evidence: moderate; figure $4 \mathrm{E}$ ), nor any effects from subgroups.

\section{Secondary vascular events}

One study randomised 70 patients with non-cardioembolic stroke to a 24-week programme of exercise including salt reduction and dietary education or to usual care with health education. ${ }^{39}$ It was 


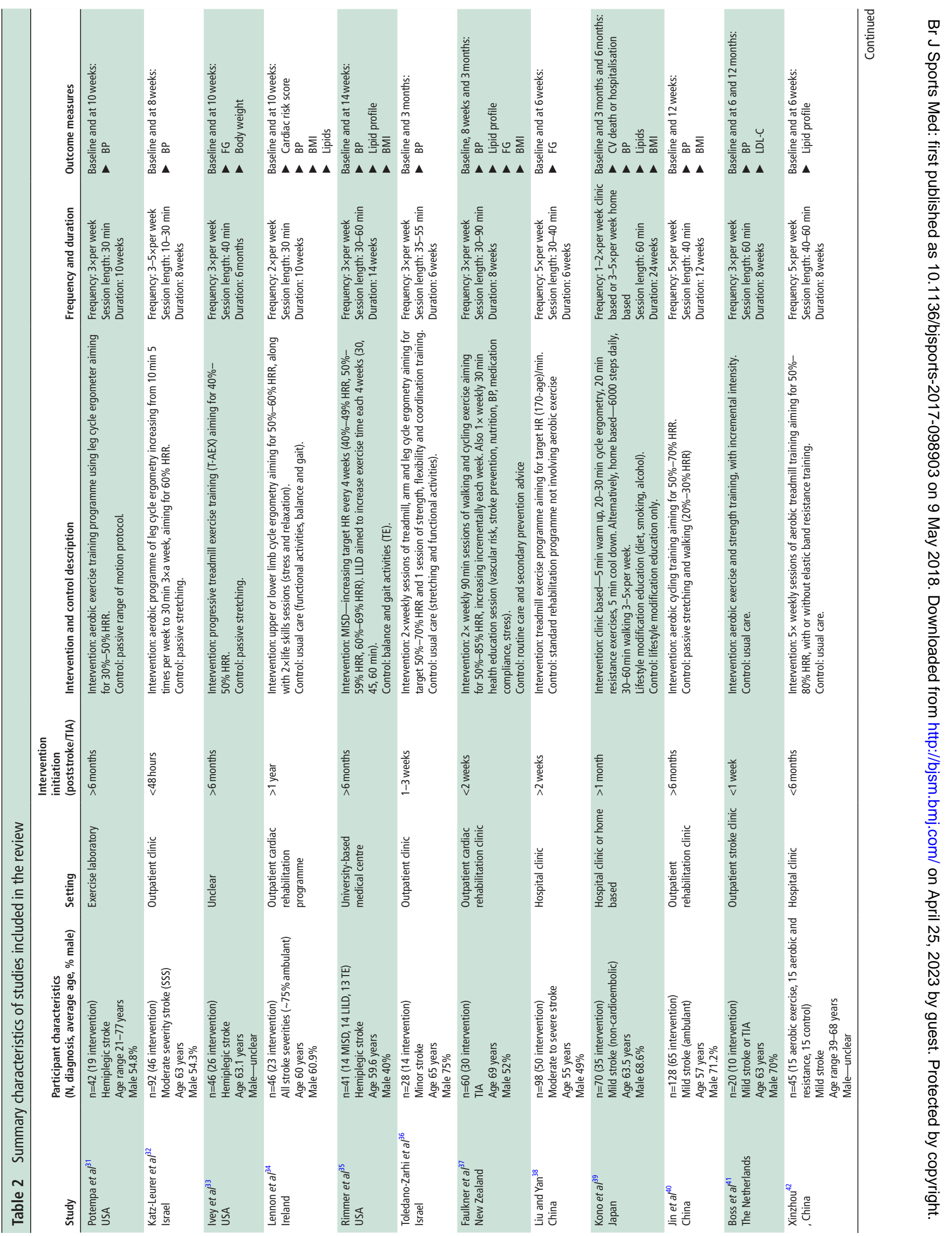




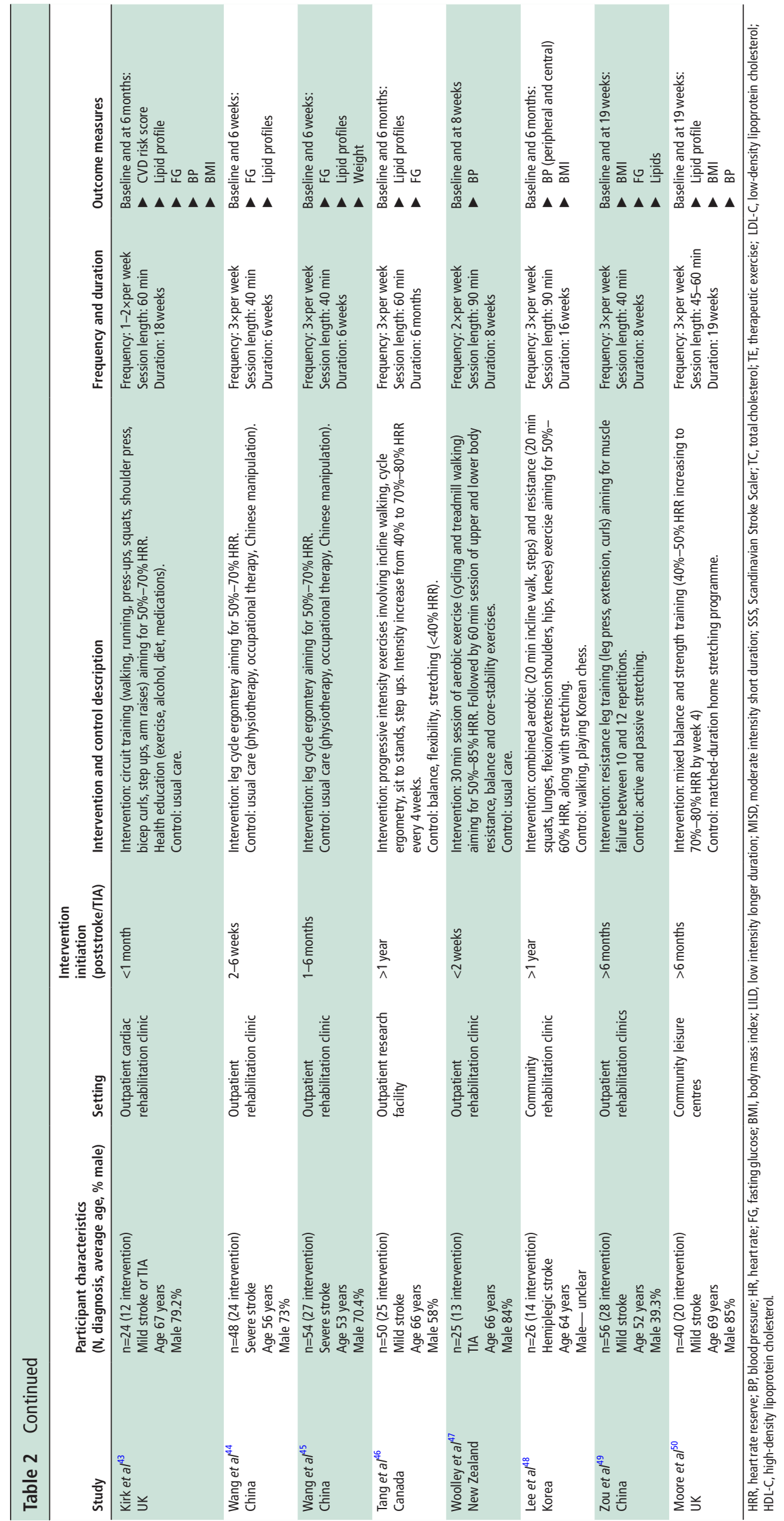

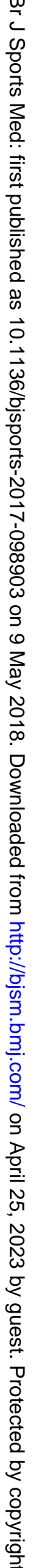


A

\begin{tabular}{|c|c|c|c|}
\hline Study or Subaroup & $\begin{array}{r}\text { Exper } \\
\text { Mean ImmHal }\end{array}$ & $\begin{array}{l}\text { mental } \\
\text { SD [mmHal] }\end{array}$ & Toto \\
\hline 1.2.1 Without subgroups & & & \\
\hline Faulkner, James 2013 & -11.3 & 16.37448 & \\
\hline Jin et al 2013 & -1 & 12.32233 & \\
\hline Katz-Leurer et al 2003 & -5.7 & 29.7 & \\
\hline Kirk, Hayden 2014 & -4.58 & 16.2 & \\
\hline Kono, Yuji 2013 & -13 & 18.36576 & \\
\hline Lee, Yong Hee 2015 & -4.93 & 9.251746 & \\
\hline Lennon Olive et al. 2008 & -1.1 & 23.03018 & \\
\hline Moore, Sarah A 2015 & 6 & 26.83983 & \\
\hline Potempa et al 1995 & -7.1 & 2.954811 & \\
\hline Rimmer, James H 2009 et al. 2009 & -10.3 & 17.65687 & \\
\hline Toledano-Zarhi, Adi et al. 2011 & -3.2 & 12.46956 & \\
\hline Woolley, B 2014 & -7 & 9 & \\
\hline Subtotal $(95 \% \mathrm{Cl})$ & & & \\
\hline
\end{tabular}

Control

Mean Difference

Mean Difference

$\begin{array}{rrrr}m m H a l & \text { SD immHal } & \text { Total } & \text { Weiaht } \\ -1.5 & 16.37448 & 35 & 1.4 \% \\ -0.8 & 12.32233 & 63 & 3.3 \% \\ 2.8 & 49.6 & 44 & 0.3 \% \\ -2.4 & 11.8 & 12 & 0.7 \% \\ 2.1 & 18.36576 & 30 & 1.0 \% \\ -1.83 & 8.543109 & 20 & 2.3 \% \\ -1.7 & 23.03018 & 23 & 0.5 \% \\ 2 & 13.00755 & 12 & 0.3 \% \\ -4.5 & 3.321053 & 23 & 6.6 \% \\ -2.5 & 5.986417 & 13 & 0.9 \% \\ 0 & 12.41008 & 14 & 1.0 \% \\ 2 & 9 & 12 & 1.6 \% \\ & & 301 & 20.0 \%\end{array}$

Random. $95 \% \mathrm{Cl}[\mathrm{mmHal}$ IV. Random, $95 \% \mathrm{Cl}[\mathrm{mmHa}]$

B

1.2.2 Interventions started $<6$ months

Faulkner, James 2013

Katz-Leurer et al 2003

Kirk, Hayden 2014

Kono, Yuji 2013

Toledano-Zarni, Adi et al. 2011

Woolley, B 2014

$\begin{array}{rrr}-11.3 & 16.37448 & 35 \\ -5.7 & 29.7 & 46 \\ -4.58 & 16.2 & 12 \\ -13 & 18.36576 & 30 \\ -3.2 & 12.46956 & 14 \\ -7 & 9 & 14 \\ & & 151\end{array}$

$\begin{array}{rrrr}-1.5 & 16.37448 & 35 & 1.4 \% \\ 2.8 & 49.6 & 44 & 0.3 \% \\ -2.4 & 11.8 & 12 & 0.7 \% \\ 2.1 & 18.36576 & 30 & 1.0 \% \\ 0 & 12.41008 & 14 & 1.0 \% \\ 2 & 9 & 12 & 1.6 \% \\ & & 147 & 6.0 \%\end{array}$

Heterogeneity, $\mathrm{Tau}^{2}=0.00 ; \mathrm{Ch}^{2}=4.53, \mathrm{df}=5(P=0.48) ; \mathrm{I}^{2}=0 \%$

Test for overall effect $Z=4.46(P<0.00001)$

1.2.3 interventions started $>6$ months

Jin et al 2013

Lee, Yong Hee 2015

Lennon Olive et al. 2008

Moore, Sarah A 2015

Potempa et al 1995

Rimmer, James H 2009 et al. 2009

Subtotal $(95 \% \mathrm{Cl})$

Heterogeneity $\mathrm{TaU}^{2}=0.00 ; \mathrm{Ch}^{2}=3.07, \mathrm{df}=5(\mathrm{P}=0.69) ; \mathrm{I}^{2}=0 \%$

$\begin{array}{rrr}-1 & 12.32233 & 65 \\ -4.93 & 9.251746 & 20 \\ -1.1 & 23.03018 & 23 \\ 6 & 26.83983 & 13 \\ -7.1 & 2.954811 & 19 \\ -10.3 & 17.65687 & 14 \\ & & 154\end{array}$

Test for overall effect $Z=2.83(P=0.005)$

C

1.2.6 Exercise only

Jin et al 2013

Katz-Leurer et al 2003

Lee, Yong Hee 2015

Moore, Sarah A 2015

Potempa et al 1995

Rimmer, James H 2009 et al. 2009

Toledano-Zarhi, Adi et al. 2011

Woolley, B 2014

Subtotal $(95 \% \mathrm{Cl})$

Heterogeneity. $\mathrm{Tau}^{2}=0.00 ; \mathrm{Chi}^{2}=6.65, \mathrm{df}=7(\mathrm{P}=0.47) ; \mathrm{P}^{2}=0 \%$

$\begin{array}{rrr}-1 & 12.32233 & 65 \\ -5.7 & 29.7 & 46 \\ -4.93 & 9.251746 & 20 \\ 6 & 26.83983 & 13 \\ -7.1 & 2.954811 & 19 \\ -10.3 & 17.65687 & 14 \\ -3.2 & 12.46956 & 14 \\ -7 & 9 & 14 \\ & & 205\end{array}$

Test for overall effect $Z=3.51(P=0.0004)$

1.2.7 Exercise and education

Faulkner, James 2013

Kirk, Hayden 2014

Kono, Yuji 2013

Lennon Olive et al. 2008

$\begin{array}{lll}11.3 & 16.37448 & 35\end{array}$

$\begin{array}{rrr}-13 & 18.2 & 12\end{array}$

$\begin{array}{rrr}-13 & 18.36576 & 30 \\ -1.1 & 23.03018 & 23\end{array}$

Subtotal $(95 \% \mathrm{Cl})$

$(P=0.17) ; 1^{2}=40 \%$

Heterogeneity. Tau $^{2}=17.83 ; \mathrm{Chi}^{2}=5.04, \mathrm{df}=3(P=0.17) ; 1^{2}=40 \%$
Test for overall effect. $Z=2.34(P=0.02)$

$-9.80[-17.47,-2.13]$

$-0.20[-4.47,4.07]$

$-8.50[-25.48,8.48]$

$-2.18[-13.52,9.16]$

$-15.10[-24.39,-5.81]$

$-3.10[-8.62,2.42]$

$0.60[-12.71,13.91]$

$4.00[-12.34,20.34]$

$-2.60[-4.50,-0.70]$

$-7.80[-17.60,2.00]$

$-3.20[-12.42,6.02]$

$-9.00[-15.94,-2.06]$

$-4.30[-6.77,-1.83]$

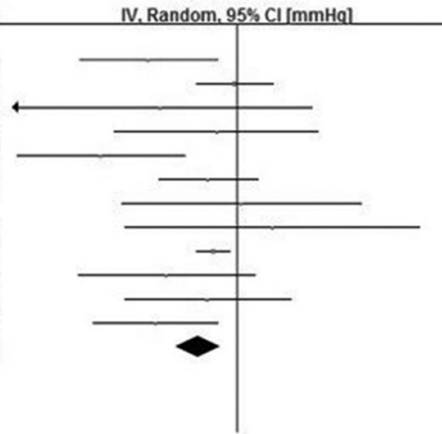

D

1.2.8 TIA / non-disabling stroke

Faulkner, James 2013

Jin et al 2013

Kirk, Hayden 2014

Kono, Yuji 2013

Lee, Yong Hee 2015

Moore, Sarah A 2015

Rimmer, James H 2009 et al. 2009

Toledano-Zarhi, Adi et al. 2011

Woolley, B 2014

$\begin{array}{rr}-11.3 & 16.37448 \\ -1 & 12.32233 \\ -4.58 & 16.2 \\ -13 & 18.36576 \\ -4.93 & 9.251746 \\ 6 & 26.83983 \\ -10.3 & 17.65687 \\ -3.2 & 12.46956 \\ -7 & 9\end{array}$

Heterogeneits, Tau $^{2}=11.72 ; \mathrm{Chi}^{2}=14.30, \mathrm{df}=8(P=0.07) ; \mathrm{I}^{2}=44 \%$

Heterogeneity. Tau $^{2}=11.72 ; \mathrm{Chi}^{2}=14.30$
Test for overall effect: $Z=2.96(P=0.003)$

1.2.9 More disabling stroke

Katz-Leurer et al 2003

Lennon Olive et al. 2008

Potempa et al 1995

Subtotal $(95 \% \mathrm{Cl})$

Heterogeneity, $\mathrm{Ta}^{2}=0.00 ; \mathrm{Chi}^{2}=0.69, \mathrm{df}=2(P=0.71) ; \mathrm{P}^{2}=0 \%$

$-9.80[-17.47,-2.13$

$-8.50[-25.48,8.48]$

$-2.18[-13.52,9.16]$

$15.10[-24.39,-5.81]$

$-3.20[-12.42,6.02]$

$-9.00[-15.94,-2.06]$

$8.46[-12.18,-4.75]$

$\begin{array}{rlll}-0.8 & 12.32233 & 63 & 3.3 \% \\ -1.83 & 8.543109 & 20 & 2.3 \% \\ -1.7 & 23.03018 & 23 & 0.5 \% \\ 2 & 13.00755 & 12 & 0.3 \% \\ -4.5 & 3.321053 & 23 & 6.6 \% \\ -2.5 & 5.986417 & 13 & 0.9 \%\end{array}$

$-0.20[-4.47,4.07]$ $-3.10[-8.62,2.42]$ $0.60[-12.71,13.91]$ $4.00[-12.34,20.34]$

$-2.60[-4.50,-0.70]$

$7.70617 .60,2001$

$-2.33[-3.94,-0.72]$

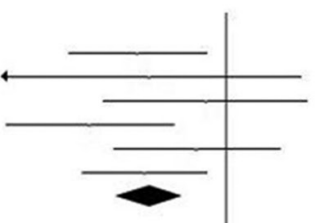

$-0.20[-4.47,4.07]$

$-8.50[-25.48,8.48]$

$-3.10[-8.62,2.42]$

$4.00[-12.34,20.34]$

$-2.60[-4.50,-0.70]$

$-7.80[-17.60,2.00]$

$-3.20+12.42,6.02]$

$-9.00[-15.94,-2.06]$

$-2.78[-4.33,-1.23]$
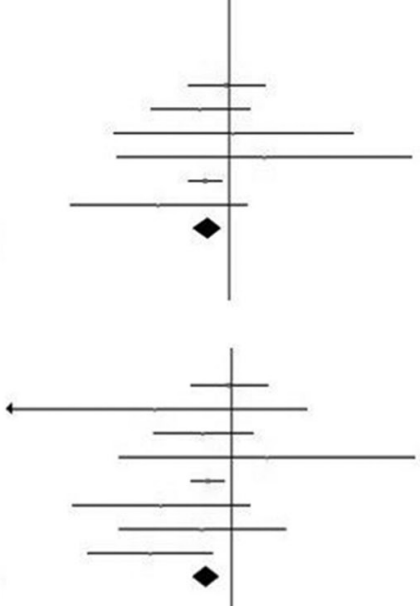

Test for overall effect $Z=2.74(\mathrm{P}=0.006)$

$\begin{array}{rrrr}-1.5 & 16.37448 & 35 & 1.4 \% \\ -2.4 & 11.8 & 12 & 0.7 \% \\ 2.1 & 18.36576 & 30 & 1.0 \% \\ -1.7 & 23.03018 & 23 & 0.5 \% \\ & & 100 & 3.6 \%\end{array}$

$-9.80[-17,47,-2.13]$

$-2.18+13.52,9.16]$

$-15.10[-24.39,-5.81]$

$0.60[-12.71,13.91]$

$-7.81[-14.34,-1.28]$
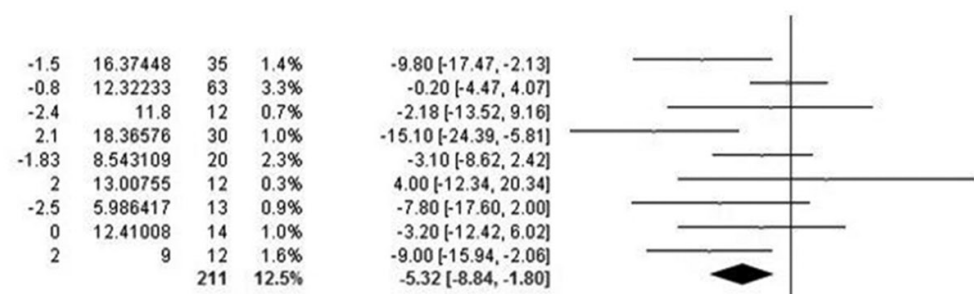

$-5.32[-8.84,-1.80]$

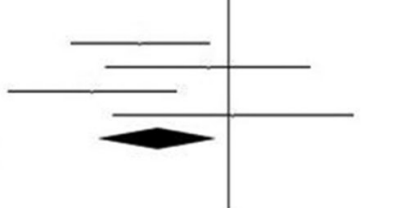

$\begin{array}{rrrr}2.8 & 49.6 & 44 & 0.3 \% \\ -1.7 & 23.03018 & 23 & 0.5 \% \\ -4.5 & 3.321053 & 23 & 6.6 \% \\ & & 90 & 7.5 \%\end{array}$
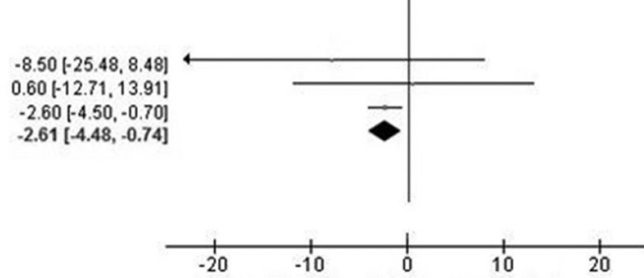

Favours [experimental] Favours [control]

Figure 2 Forest plots of the effects of exercise vs control interventions on (A) systolic blood pressure, including effects of (B) early ( $<6$ months) vs late initiation (>6 months), (C) inclusion of an educational component and (D) population condition (stroke vs transient ischaemic attack). 
Exercise

Control

Mean Difference

Mean Difference Mean ImmHal SD ImmHal Total Mean ImmHal SDImmHal Total Weight IV, Random, $95 \%$ CI ImmHal

IV. Random, $95 \% \mathrm{Cl}[\mathrm{mmHa}$

Study or Subaroup 1.3.1 Without Subgroups Faulkner, James 2013 Jin et al 2013

Katz-Leurer et al 2003

Kirk, Hayden 2014

Kono, Yuji 2013

Lee, Yong Hee 2015

Lennon Olive et al. 2008

Moore, Sarah A 2015

Potempa et al 1995

Rimmer, James H 2009 et al. 2009

Toledano-Zarhi, Adi et al. 2011

Woolley, B 2014

$\begin{array}{rr}-2.1 & 5.966155 \\ -0.8 & 10.29402 \\ -3.2 & 29.8 \\ -4.41 & 8.3 \\ -6.3 & 5.838121 \\ -2.5 & 4.371126 \\ 1.7 & 8.33894 \\ -3 & 13.13824 \\ -4 & 1.484924 \\ -8.7 & 8.450822 \\ -1.9 & 146.0900551 \\ -9 & 15\end{array}$

Heterogeneits: Tau $^{2}=6.92 ; \mathrm{Chi}^{2}=33.85, \mathrm{df}=11(\mathrm{P}=0.0004) ; \mathrm{P}^{2}=68 \%$

Test for overall effect: $Z=2.38(P=0.02)$

B

1.3.2 Interventions started $<6$ months

$\begin{array}{lrrr}\text { Faulkner, James 2013 } & -2.1 & 5.966155 & 30 \\ \text { Katz-Leurer et al 2003 } & -3.2 & 29.8 & \\ \text { Kirk, Hayden 2014 } & -4.41 & 8.3 & 12 \\ \text { Kono, Yuji 2013 } & -6.3 & 5.838121 & 35 \\ \text { Toledano-Zarmi, Adi et al. 2011 } & -1.9 & 146.0900551 & 1 \\ \text { Woolley, B 2014 } & -9 & 15 & 13 \\ \text { Subtotal (95\% Cl) } & & & 150\end{array}$

Subtotal $(95 \% \mathrm{Cl})$

$\begin{array}{rrrr}0.2 & 6.514599 & 30 & 2.6 \% \\ 0.8 & 10.29402 & 63 & 2.3 \% \\ 2.3 & 17.94 & 44 & 0.5 \% \\ -5 & 8.7 & 12 & 1.0 \% \\ 1.5 & 5.838121 & 35 & 2.8 \% \\ 1 & 4.371126 & 12 & 2.4 \% \\ 0.2 & 8.33894 & 23 & 1.7 \% \\ 5 & 10.14227 & 20 & 0.9 \% \\ 4.7 & 1.6 & 23 & 4.1 \% \\ 0.5 & 11.21797 & 13 & 0.9 \% \\ 1.8 & 146.0900551 & 14 & 0.0 \% \\ 1 & 3 & 12 & 0.7 \% \\ & & 301 & 20.0 \%\end{array}$

$-1901500,120$

$-1.60[-5.17,1.97)$

$-0.90[-11.01,9.21]$

$0.59[-6.21,7.39]$

$-4.80[-7.54,-2.06]$

$1.50[-3.32,6.32$

$-8.00[-15.27,-0.73]$

$0.70[-0.23,1.63]$

$-8.20+15.74,-0.69$

$-0.10[-108.32,108.12]$

$10.00[-18.33,-1.67]$
$-2.58[-4.71,-0.46]$

列

Test for overall effect $Z=2.92(P=0.003)$

$\begin{array}{lrr}\text { 1.3.3 interventions started > } 6 \text { months } & & \\ \text { Jin et al } 2013 & -0.8 & 10.29402 \\ \text { Lee, Yong Hee 2015 } & -2.5 & 4.371126 \\ \text { Lennon Olive et al. 2008 } & 1.7 & 8.33894 \\ \text { Moore, Sarah A 2015 } & -3 & 13.13824 \\ \text { Potempa et al 1995 } & -4 & 1.484924 \\ \text { Rimmer, James H 2009 et al. 2009 } & -8.7 & 8.450822 \\ \text { Subtotal (95\% CI) } & & \end{array}$

Subtotal $(95 \% \mathrm{Cl})$

Test for overall effect $Z=1.49(P=0.14)$

C

1.3.6 Exercise only

Jin et al 2013

Katz-Leurer et al 2003

Lee, Yong Hee 2015

Moore, Sarah A 2015

Potempa et al 1995

Rimmer, James $\mathrm{H} 2009$ et al. 2009

Toledano-Zarhi, Adi et al. 2011

Woolley, B 2014

Subtotal $(95 \% \mathrm{Cl})$

Heterogeneity Tau $^{2}=9.49 ; \mathrm{Chi}^{2}=21.82$, df $=7(P=0.003) ; \mathrm{P}^{\mathrm{P}}=68 \%$

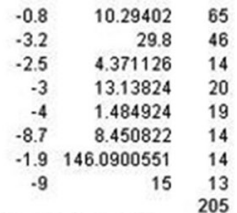

D

1.3.8 TIA / non-disabling stroke

Faulkner, James 2013

Jin et al 2013

Kirk, Hayden 201

Kono, Yuji 2013

Lee, Yong Hee 2015

Moore, Sarah A 2015

Rimmer, James H 2009 et al. 2009

Toledano-Zarhi, Adi et al. 2011

Woolley, B 2014

$\begin{array}{rrr}-2.1 & 5.966155 & 30 \\ -4.41 & 8.3 & 12 \\ -6.3 & 5.838121 & 35 \\ 1.7 & 8.33894 & 23 \\ & & 100\end{array}$

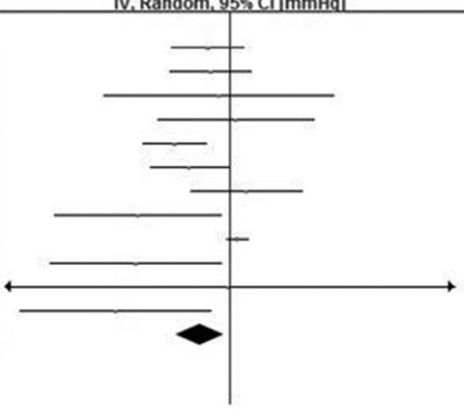

Heterogeneity Tau $^{2}=1.11 ; \mathrm{Chi}^{2}=9.63, \mathrm{df}=8(P=0.29) ;\left.\right|^{2}=17 \%$

Test for overall effect: $Z=4.20$ ( $P<0.0001)$

1.3.9 More disabling stroke

Katz-Leurer et al 2003

Lennon Olive et al. 2008

Potempa et al 1995

Subtotal $(95 \% \mathrm{Cl})$

$\begin{array}{rrr}-2.1 & 5.966155 & 30 \\ -0.8 & 10.29402 & 65 \\ -4.41 & 8.3 & 12 \\ -6.3 & 5.838121 & 35 \\ -2.5 & 4.371126 & 14 \\ -3 & 13.13824 & 20 \\ -8.7 & 8.450822 & 14 \\ -1.9 & 146.0900551 & 14 \\ -9 & 15 & 13 \\ & & 217\end{array}$

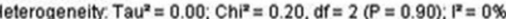

Test for overall effect: $Z=1.54(P=0.12)$
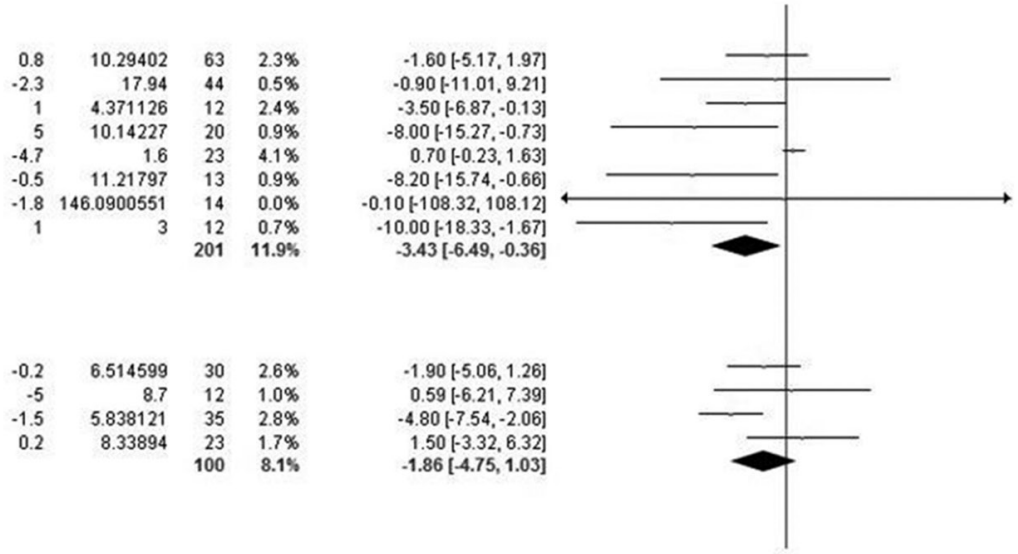

$\begin{array}{rrrr}0.8 & 10.29402 & 63 & 2.3 \% \\ 1 & 4.371126 & 12 & 2.4 \% \\ 0.2 & 8.33894 & 23 & 1.7 \% \\ 5 & 10.14227 & 20 & 0.9 \% \\ -4.7 & 1.6 & 23 & 4.1 \% \\ -0.5 & 11.21797 & 13 & 0.9 \% \\ & & 154 & 12.3 \%\end{array}$

$-1.60[-5.17,1.97]$

$-3.50[-6.87,-0.13$

$1.50[-3.32,6.32$

$-8.00[-15.27,-0.73]$

$0.70[-0.23,1.63$

$-8.20[-15.74,-0.66]$

$-2.08[-4.82,0.66]$
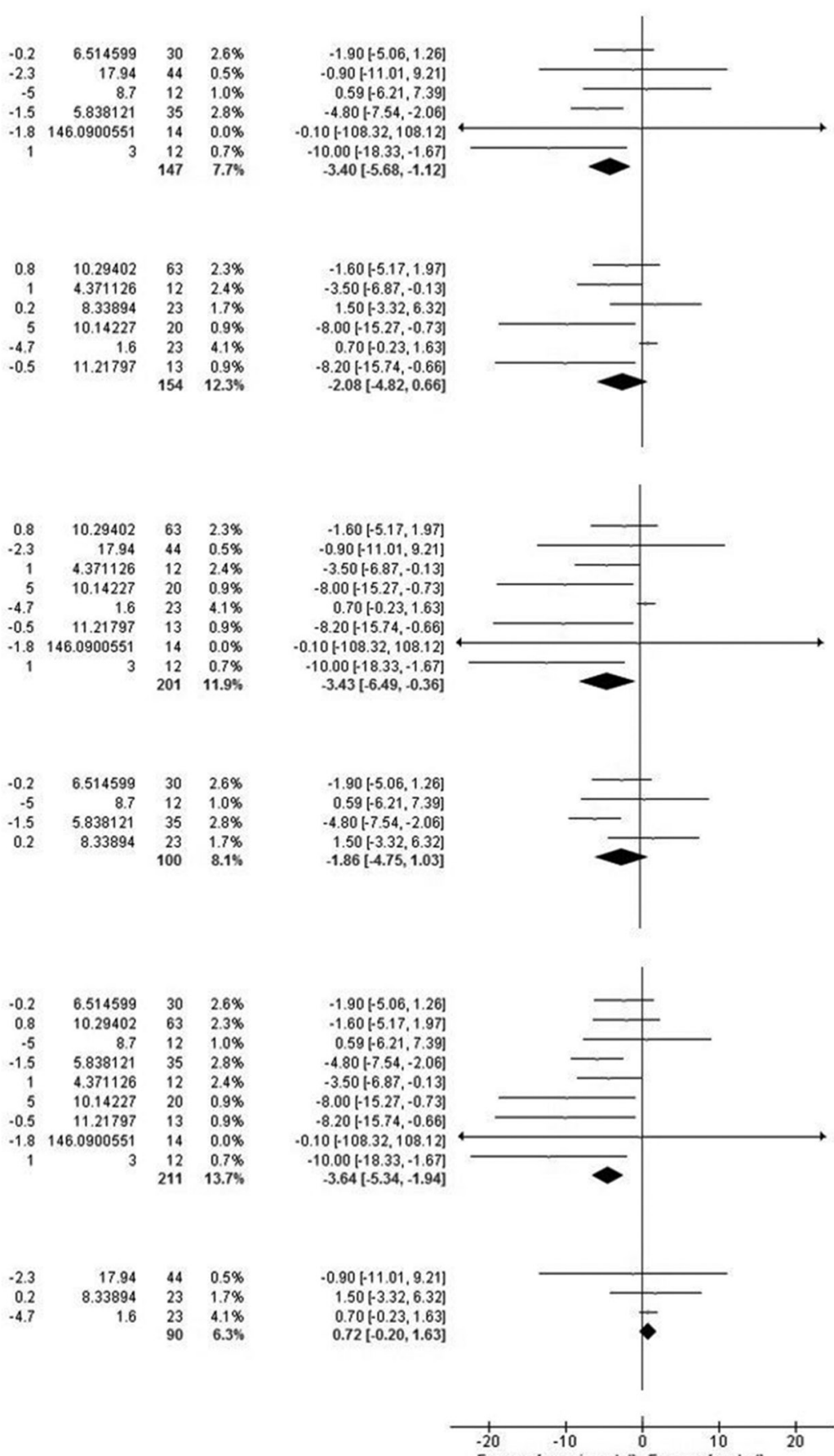

Favours [experimental] Favours [control]

Figure 3 Forest plots of the effects of exercise vs control interventions on (A) diastolic blood pressure, including effects of (B) early ( $<6$ months) vs late initiation (>6 months), (C) inclusion of an educational component, and (D) population condition (stroke vs transient ischaemic attack). 
A

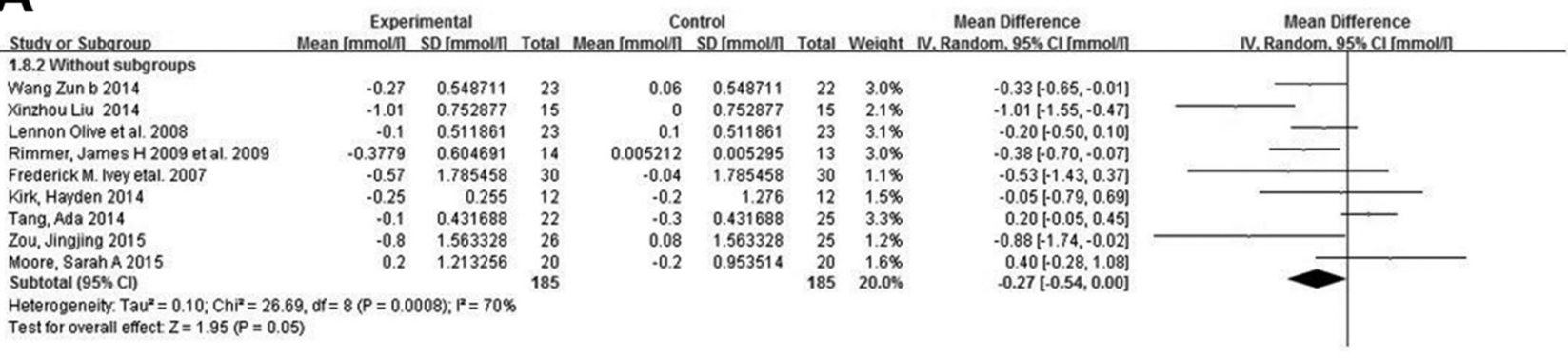

B

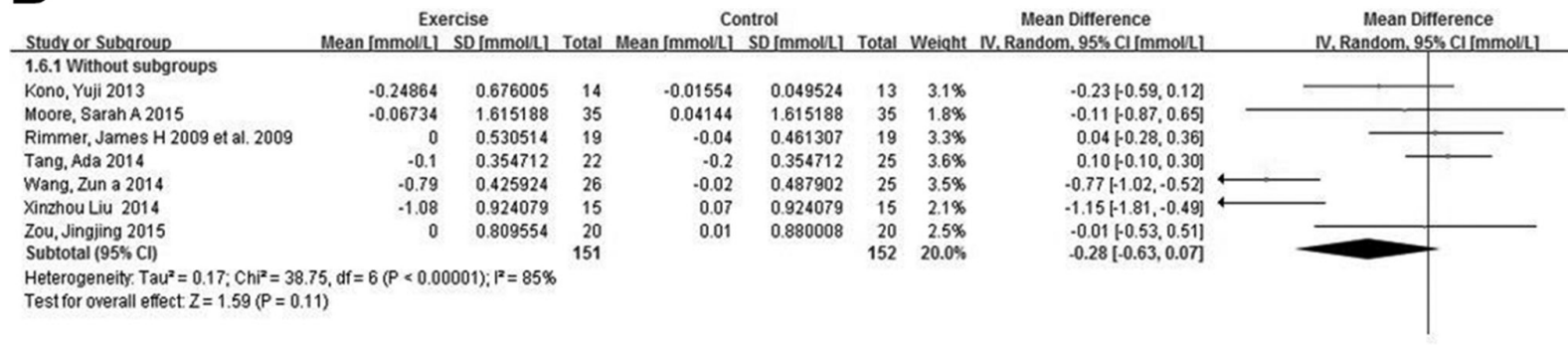

C

Study or Subaroup

Experimental

Control

Mean Difference
Random, $95 \% \mathrm{CI}$ [mmoll]

Mean Difference Studv or Subaroup Frederick M. wey etal. 2007 Kirk, Hayden 2014 Kono, Yuji 2013
Moore, Sarah A 2015

Rimmer, James $\mathrm{H} 2009$ et al. 2009

Tang, Ada 2014

Wang Zun b 2014

Xinzhou Liu 2014

Zou, Jingjing 2015

Subtotal $(95 \% \mathrm{CI})$

Mean Immolin SD Immolin To

$\begin{array}{rrr}0 & 0.193483 & 30 \\ -0.03 & 0.1 & 12 \\ 0.213709 & 0.255804 & 35 \\ 0.3 & 0.468952 & 20 \\ -0.01564 & 0.17755 & 14 \\ 0 & 1.621497 & 22 \\ 0.05 & 0.040139 & 23 \\ 0.35 & 0.521962 & 15 \\ 0.18 & 0.302006 & 26 \\ & & 197\end{array}$

Heterogeneity $\mathrm{Tau}^{2}=0.01 ; \mathrm{Chi}^{2}=35.20, \mathrm{df}=8(\mathrm{P}<0.0001): \mathrm{I}^{2}=77 \%$

Test for overall effect: $Z=1.63(P=0.10)$

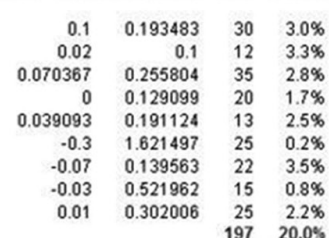

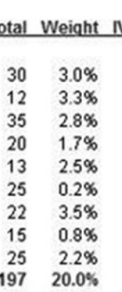
IV. Random. $95 \% \mathrm{CI}$ Immoln

D

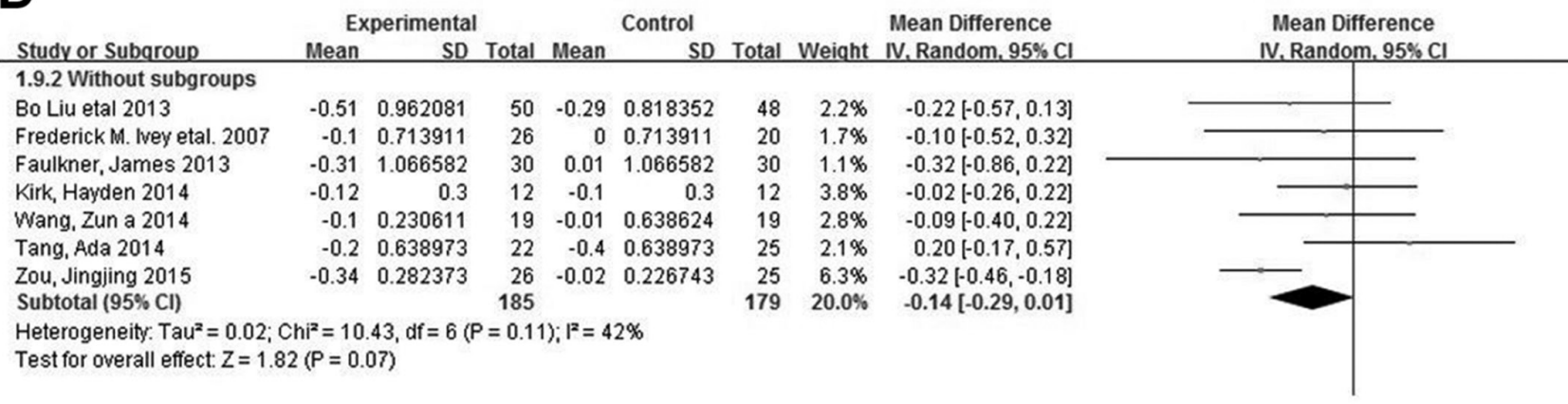

E

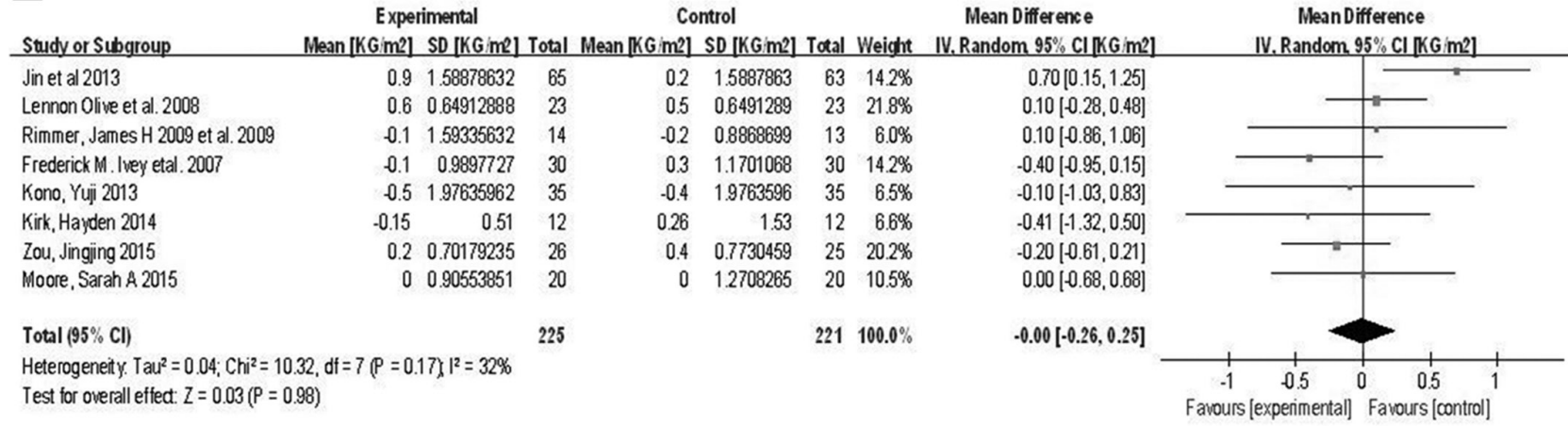

Figure 4 Forest plots of the effects of exercise vs control interventions on (A) total cholesterol, (B) low-density lipoprotein cholesterol, (C) highdensity lipoprotein cholesterol, (D) fasting glucose and (E) body mass index. 
terminated early after results showed significantly fewer hospitalisations or deaths secondary to stroke, myocardial infarction or peripheral arterial disease in the intervention group (adjusted HR $0.194,95 \%$ CI 0.121 to $0.737, \mathrm{p}=0.005)$. The intervention in this trial also resulted in greater reductions in SBP, DBP and increased levels of HDL-C.

\section{Adverse events}

Three patients from the 20 RCTs suffered adverse events. All adverse events were unrelated to the exercise interventions (see online supplementary material). One trial ${ }^{41}$ reported benign ECG abnormalities on exercise testing prior to exercise interventions, and one patient went on to have percutaneous coronary intervention for underlying CHD.

\section{DISCUSSION}

\section{Effect of exercise on vascular risk factors}

Exercise interventions can lead to clinically meaningful reductions in SBP and DBP after stroke or TIA. This is important because SBP and DBP may be equally important predictors of secondary stroke risk. ${ }^{53}$ While exercise causes immediate elevations in heart rate, cardiac output and peripheral vasodilatation through nitric oxide release, ${ }^{54}$ the long-lasting effects that may mediate blood pressure reduction include enhanced endothelial sensitivity to nitric oxide, a reduction in sympathetic tone and salt load, all ultimately reducing systemic vascular resistance. ${ }^{55}$

Antihypertensive therapy reduces the risk of secondary stroke by $24 \%$ and myocardial infarction by $21 \%$ when compared with placebo. ${ }^{56}$ A recent meta-analysis of 10 RCTs evaluating antihypertensive therapy after stroke showed that these effects occurred with reductions in SBP of $5.1 \mathrm{~mm} \mathrm{Hg}$ and DBP of $2.5 \mathrm{~mm} \mathrm{Hg}$ across a range of antihypertensive classes. ${ }^{57}$ Thus, the blood pressure reductions associated with exercise interventions in our meta-analysis (SBP $4.3 \mathrm{~mm} \mathrm{Hg}$, DBP $2.5 \mathrm{~mm} \mathrm{Hg}$ ) compare favourably with pharmacological approaches. The effects of exercise may be additive to pharmacological intervention. For example, a trial evaluating the effects of an exercise programme in patients with drug-resistant hypertension (patients already taking three antihypertensives) revealed mean SBP and DBP reductions of $6( \pm 12) \mathrm{mm} \mathrm{Hg}$ and $3( \pm 7) \mathrm{mm} \mathrm{Hg}$, respectively in the exercise group compared with controls $(\mathrm{p}=0.03) .{ }^{58}$

Exercise is associated with increased calorific expenditure, peripheral insulin sensitivity ${ }^{59}$ and enhanced muscle metabolism of cholesterol. ${ }^{60}$ Our analysis suggested that exercise may have beneficial effects on lipid profiles in patients after stroke, although heterogeneity in these data means the results should be interpreted cautiously.

Exercise programmes initiated within 6 months after stroke or TIA had greater blood pressure reductions compared with programmes initiated after 6 months. This may be because individuals are more likely to engage with behaviour modification soon after illness compared with later, often referred to as the 'sentinel event effect' as is evidenced for smoking cessation interventions following myocardial infarction. ${ }^{61}$ Early intervention may be of increased benefit after stroke and TIA as the risks of recurrence are highest within the first few months. ${ }^{62}$ However, significant reductions in SBP were still observed when interventions were started later. Therefore, promoting exercise, even in the chronic phase after stroke, may still lead to clinically important benefits. The inclusion of a health education component alongside exercise interventions also appeared beneficial. Indeed, meta-analyses of education interventions targeting diet result in clinically meaningful in blood pressure and lipids, ${ }^{63} 64$ and may be an important part of a holistic secondary stroke prevention programme.

\section{Secondary vascular events}

One study reported on vascular outcomes, reporting that exercise participants were approximately $80 \%$ less likely to suffer a vascular death or hospitalisation over a median follow-up of 2.9 years. The early initiation ( 1 month postevent), long intervention duration ( 24 weeks), inclusion of salt reduction education and the use of activity monitors (which may themselves improve overall levels of physical activity ${ }^{65}$ ) may have contributed to the observed effectiveness and the large reductions in SBP $(13.0 \mathrm{~mm} \mathrm{Hg})$ and DBP $(6.3 \mathrm{~mm} \mathrm{Hg})$. In addition, by including patients with non-cardioembolic ischaemic stroke only, the investigators may have selected a group of patients standing to benefit most from classical vascular risk reduction. Nevertheless, this was a small, single-centre study and further research to establish the link between exercise and reduced secondary vascular events remains a priority.

\section{Future research}

Another priority area for research relates to enhancing the uptake of exercise as an intervention. In the UK, despite the strong evidence base for cardiovascular mortality reduction with exercise-based cardiac rehabilitation, and its provision freely within the National Health Service, fewer than half of eligible patients take up the intervention. ${ }^{66} 67$ This may be due to a number of factors such as multimorbidity, problems with transport and poor social support. ${ }^{68}$ To extend the potential benefits of exercise, future research should evaluate facilitators and barriers to participation in exercise interventions among patients with stroke, and the role of emerging assistive technologies in enhancing intervention delivery, for example, smartphones, fitbits, tablet PCs. Future trials should also include full descriptions of intervention characteristics (ie, following the CERT) so clinicians know which types and intensities of exercise should be prescribed in clinical practice.

\section{Safety of exercise}

Patients and clinicians may have concerns about engaging in physical activity soon after stroke or TIA. Our results suggest that exercise interventions are safe to undertake in patients with stroke or TIA, even in the very early phases. A Cochrane review of 58 trials of physical fitness training for functional recovery after stroke also reported excellent safety data with no reports of severe adverse events. ${ }^{18}$

\section{Limitations}

Owing to the significant variation in the type of exercise intervention, it is difficult to ascertain the ideal design for effectiveness. The relatively small number of studies and participants precluded investigating the effects of specific components of exercise interventions (eg, single vs group sessions). However, exercise interventions initiated early rather than late, and those combined with health education appeared more effective for reducing SBP. It is still uncertain if the effects of the interventions relate to behaviour change outside of the exercise sessions. Only one study used activity monitors to link exercise with increased overall step counts. ${ }^{39}$

Since vascular risk factors were measured at the end of the study interventions, we do not know if the beneficial effects of exercise extend beyond the intervention duration. Studies of exercise in other patient cohorts have suggested a decline in 
beneficial effects soon after intervention termination, ${ }^{69}$ although data from cardiac rehabilitation studies demonstrate reductions in cardiovascular mortality and hospitalisation that persist at 12 months follow-up. ${ }^{70} 71$ The majority of participants in included trials were relatively young (average age 69 years) with mildly disabling stroke or TIA who were ambulant. This may limit the generalisability of our findings to older, more disabled patients with stroke. Our analysis suggested only modest SBP reductions among exercise studies that included more disabled patients with stroke, with no changes in DBP. These patients, who often experience greater fatigue, functional and cognitive impairment, may find it more difficult to comply with the frequency, duration and target heart rates of prescribed exercise that lead to beneficial effects.

The quality of evidence (GRADE) for the outcomes ranged from moderate to very low, primarily due to inconsistency and imprecision of results. All of the included studies were small pilot trials, none powered independently to detect significant differences in any of the vascular risk factors measured. These small studies may also be subject to inherent exaggeration of effect sizes due to variations in methodological quality and heterogeneity of patient cohorts. Although we performed funnel plots for publication bias, we did not search grey literature nor included manuscripts outside of the English and Chinese language. Therefore, there is a risk of publication and language bias.

Our results suggest that clinicians managing patients with stroke or TIA should recommend aerobic exercise (eg, brisk walking, cycling, stepping), aiming for target heart rate reserves of between $50 \%$ and $70 \%$, for around $150-180 \mathrm{~min}$ per week (divided over 2-3 sessions). Exercise sessions should be prioritised to start early (ie, within 6 months) after stroke or TIA. One way to deliver the suggested aerobic exercise interventions in combination with health education, may be to refer to existing local phase III/IV cardiac rehabilitation services, some of which across the UK are already accepting stroke referrals. ${ }^{43}$ However, in order for this to become an established service, high-quality RCTs, recruiting larger, less selected groups of stroke survivors, and incorporating longer follow-up, are desperately needed to investigate the effects of exercise on cardiovascular events and mortality.

\section{CONCLUSION}

Exercise interventions poststroke and TIA are safe and contributed to clinically meaningful reductions in blood pressure, the strongest modifiable predictor of secondary stroke. This was most pronounced among interventions initiated within

\section{What is already known}

- Exercise after stroke has been shown to improve walking speed and balance, but its effect on secondary vascular risk factors is less well studied.

\section{What this study adds}

- Exercise programmes after stroke or TIA are safe and result in clinically meaningful reductions in systolic and diastolic blood pressure.

- The beneficial effects of exercise programmes are enhanced if started within 6 months of stroke or TIA and incorporate health education.
6 months of the index event and those incorporating health education. There were also beneficial effects of exercise for lipid profiles.

Contributors ANA conceived the review. ANA and CW led the research team in undertaking the systematic review and meta-analysis. ANA, CW and MS were involved in study selection and quality appraisal. All authors were involved in manuscript development.

Funding This research received no specific grant from any funding agency in the public, commercial or not-for-profit sectors.

Competing interests None declared.

Patient consent Not required.

Provenance and peer review Not commissioned; externally peer reviewed.

(c) Article author(s) (or their employer(s) unless otherwise stated in the text of the article) 2019. All rights reserved. No commercial use is permitted unless otherwise expressly granted.

\section{REFERENCES}

1 World Health Organisation. The top 10 causes of death. 2015. http://www.who.int/ mediacentre/factsheets/fs310/en/ (accessed Oct 2017).

2 Grysiewicz RA, Thomas K, Pandey DK. Epidemiology of ischemic and hemorrhagic stroke: incidence, prevalence, mortality, and risk factors. Neurol Clin 2008;26:871-95

3 Slot KB, Berge E, Dorman P, et al. Impact of functional status at six months on long term survival in patients with ischaemic stroke: prospective cohort studies. BMJ 2008;336:376-9.

4 Saka O, McGuire A, Wolfe C. Cost of stroke in the United Kingdom. Age Ageing 2009;38:27-32.

5 European Cardiovascular Disease Statistics. European Heart Network, Brussels. 2012. https://www.escardio.org/static_file/Escardio/Press-media/press-releases/2013/EUcardiovascular-disease-statistics-2012.pdf (accessed Oct 2017).

6 Mozaffarian D, Benjamin EJ, Go AS, et al. Heart Disease and Stroke Statistics-2016 Update: A Report From the American Heart Association. Circulation 2016;133.

7 Hankey GJ, Jamrozik K, Broadhurst RJ, et al. Long-term disability after first-ever stroke and related prognostic factors in the Perth Community Stroke Study, 1989-1990. Stroke 2002;33:1034-40.

8 Baigent C, Blackwell L, Collins R, et al. Aspirin in the primary and secondary prevention of vascular disease: collaborative meta-analysis of individual participant data from randomised trials. Lancet 2009;373:1849-60.

9 Yusuf S, Diener HC, Sacco RL, et al. Telmisartan to prevent recurrent stroke and cardiovascular events. N Engl J Med 2008;359:1225-37.

10 Amarenco P, Benavente 0, Goldstein LB, et al. Results of the Stroke Prevention by Aggressive Reduction in Cholesterol Levels (SPARCL) trial by stroke subtypes. Stroke 2009:40:1405-9.

11 Musialek P, Hopf-Jensen S. Commentary: carotid artery revascularization for stroke prevention: a new Era. J Endovasc Ther 2017;24.

12 Lee CD, Folsom AR, Blair SN. Physical activity and stroke risk: a meta-analysis. Stroke 2003;34:2475-81.

13 British Heart Foundation. Physical activity statistics 2015. 2015. https://www.bhf.org. uk/-/media/files/./bhf_physical-activity-statistics-2015feb.pdf (accessed Oct 2017).

14 Kernan WN, Ovbiagele B, Black HR, et al. Guidelines for the prevention of stroke in patients with stroke and transient ischemic attack: a guideline for healthcare professionals from the American Heart Association/American Stroke Association. Stroke 2014;45:2160-236.

15 Lawler PR, Filion KB, Eisenberg MJ. Efficacy of exercise-based cardiac rehabilitation post-myocardial infarction: a systematic review and meta-analysis of randomized controlled trials. Am Heart J 2011;162:571-84.

16 Silberman A, Banthia R, Estay IS, et al. The effectiveness and efficacy of an intensive cardiac rehabilitation program in 24 sites. Am J Health Promot 2010;24:260-6.

17 British Heart Foundation. National Audit of Cardiac Rehabilitation. Annual report 2011-12. 2012. www.cardiacrehabilitation.org (accessed Jun 2017).

18 Saunders D, Sanderson M, Hayes S, et al. Physical fitness training for stroke survivors. Cochrane Database of Systematic Reviews 2016;3. CD003316.

19 Mackay-Lyons M, Thornton M, Ruggles T, et al. Non-pharmacological interventions for preventing secondary vascular events after stroke or transient ischaemic attack. Cochrane Database of Systematic Reviews 2013;2. CD008656.

20 Lennon 0, Galvin R, Smith K, et al. Lifestyle interventions for secondary disease prevention in stroke and transient ischaemic attack: a systematic review. Eur J Prev Cardiol 2014;21:1026-39.

21 Moher D, Liberati A, Tetzlaff J, et al. Preferred reporting items for systematic reviews and meta-analyses: the PRISMA statement. Int J Surg 2010;8:336-41.

22 Higgins J, Green S. Cochrane Handbook for systematic reviews of interventions version 5.1.0. The Cochrane Collaboration 2011 http://handbook.cochrane.org/ (accessed Jun 2017). 
23 WHO MONICA Project Investigators. The World Health Organisation MONICA Project (Monitoring trends and determinants in cardiovascular disease). Journal of Clinical Epidemiology 1988;41:105-14.

24 Slade SC, Dionne CE, Underwood M, et al. Consensus on Exercise Reporting Template (CERT): modified delphi study. Phys Ther 2016;96:1514-24.

25 Higgins J, Altman D, Gøtzsche P, et al. Cochrane Bias methods group; cochrane statistical methods group. The Cochrane Collaboration's tool for assessing risk of bias in randomised controlled trials. BMJ 2011;343:d5928.

26 Ryan R, Hill S. How to GRADE the quality of the evidence. Cochrane Consumers and Communication Group 2016. http://cccrg.cochrane.org/author-resources.

27 Furlan AD, Pennick V, Bombardier C, et al. 2009 updated method guidelines for systematic reviews in the Cochrane Back Review Group. Spine 2009;34:1929-41.

28 Thorlund K, Imberger G, Johnston BC, et al. Evolution of heterogeneity (I2) estimates and their 95\% confidence intervals in large meta-analyses. PLOS One 2012;7:e39471.

29 Egger M, Davey Smith G, Schneider M, et al. Bias in meta-analysis detected by a simple, graphical test. BMJ 1997;315:629-34.

30 Begg CB, Mazumdar M. Operating characteristics of a rank correlation test for publication bias. Biometrics 1994;50:1088-101.

31 Potempa K, Lopez M, Braun LT, et al. Physiological outcomes of aerobic exercise training in hemiparetic stroke patients. Stroke 1995;26:101-5.

32 Katz-Leurer M, Shochina M, Carmeli E, et al. The influence of early aerobic training on the functional capacity in patients with cerebrovascular accident at the subacute stage. Arch Phys Med Rehabil 2003;84:1609-14.

33 Ivey FM, Ryan AS, Hafer-Macko CE, et al. Treadmill aerobic training improves glucose tolerance and indices of insulin sensitivity in disabled stroke survivors: a preliminary report. Stroke 2007:38:2752-8.

34 Lennon 0, Carey A, Gaffney N, et al. A pilot randomized controlled trial to evaluate the benefit of the cardiac rehabilitation paradigm for the non-acute ischaemic stroke population. Clin Rehabil 2008;22:125-33.

35 Rimmer JH, Rauworth $A E$, Wang EC, et al. A preliminary study to examine the effects of aerobic and therapeutic (nonaerobic) exercise on cardiorespiratory fitness and coronary risk reduction in stroke survivors. Arch Phys Med Rehabil 2009;90:407-12.

36 Toledano-Zarhi A, Tanne D, Carmeli E, et al. Feasibility, safety and efficacy of an early aerobic rehabilitation program for patients after minor ischemic stroke: A pilot randomized controlled trial. NeuroRehabilitation 2011;28:85-90.

37 Faulkner J, Lambrick D, Woolley B, et al. Effects of early exercise engagement on vascular risk in patients with transient ischemic attack and nondisabling stroke. $J$ Stroke Cerebrovasc Dis 2013;22:e388-96.

38 Liu Bo, Yan H. Effect of treadmill walking training on stroke patients with impaired glucose tolerance. Journal of Ningxia Medical University 2013;909:35-8.

39 Kono Y, Yamada S, Yamaguchi J, et al. Secondary prevention of new vascular events with lifestyle intervention in patients with noncardioembolic mild ischemic stroke: a single-center randomized controlled trial. Cerebrovasc Dis 2013;36:88-97.

40 Jin $\mathrm{H}$, Jiang $\mathrm{Y}$, Wei Q, et al. Effects of aerobic cycling training on cardiovascular fitness and heart rate recovery in patients with chronic stroke. NeuroRehabilitation 2013:32:327-35.

41 Boss HM, Van Schaik SM, Deijle IA, et al. Safety and feasibility of post-stroke care and exercise after minor ischemic stroke or transient ischemic attack: MotiveS \& MovelT. NeuroRehabilitation 2014;34:401-7.

42 Liu X. Effects of Aerobic associated resistance exercise on the ability of exercise and quality of life in patients with stroke[D]: XiangYa School of Medicine, CSU, 2013.

43 Kirk H, Kersten P, Crawford P, et al. The cardiac model of rehabilitation for reducing cardiovascular risk factors post transient ischaemic attack and stroke: a randomized controlled trial. Clin Rehabil 2014;28:339-49.

44 Wang Z, Wang L, Fan $\mathrm{H}$, et al. Adapted low intensity ergometer aerobic training for early and severely impaired stroke survivors: a pilot randomized controlled trial to explore its feasibility and efficacy. J Phys Ther Sci 2014;26:1449-54.

45 Wang Z, Wang L, Fan H, et al. Effect of low-intensity ergometer aerobic training on glucose tolerance in severely impaired nondiabetic stroke patients. Journal of Stroke and Cerebrovascular Diseases 2014;23:e187-e193.

46 Tang A, Eng JJ, Krassioukov AV, et al. Exercise-induced changes in cardiovascular function after stroke: a randomized controlled trial. Int J Stroke 2014;9:883-9.

47 Woolley B, Stoner L, Lark S, et al. Effect of early exercise engagement on arterial stiffness in patients diagnosed with a transient ischaemic attack. J Hum Hypertens 2015;29:87-91
48 Lee YH, Park SH, Yoon ES, et al. Effects of combined aerobic and resistance exercise on central arterial stiffness and gait velocity in patients with chronic poststroke hemiparesis. Am J Phys Med Rehabil 2015;94:687-95.

49 Zou J, Wang Z, Qu Q, et al. Resistance training improves hyperglycemia and dyslipidemia, highly prevalent among nonelderly, nondiabetic, chronically disabled stroke patients. Arch Phys Med Rehabil 2015;96:1291-6.

50 Moore SA, Hallsworth K, Jakovljevic DG, et al. Effects of community exercise therapy on metabolic, brain, physical, and cognitive function following stroke: a randomized controlled pilot trial. Neurorehabil Neural Repair 2015;29:623-35.

51 Fulcher J, O'Connell R, Voysey M, et al. Efficacy and safety of LDL-lowering therapy among men and women: meta-analysis of individual data from 174,000 participants in 27 randomised trials. Lancet 2015;385:1397-405.

52 Sacco RL, Benson RT, Kargman DE, et al. High-density lipoprotein cholesterol and ischemic stroke in the elderly: the Northern Manhattan Stroke Study. JAMA 2001:285:2729-35.

53 Cornelissen VA, Fagard RH. Effects of endurance training on blood pressure, blood pressure-regulating mechanisms, and cardiovascular risk factors. Hypertension 2005;46:667-75

54 Monteiro M, Filho S. Physical exercise and blood pressure control. Brazilian Journal of Sporting Medicine 2004;10:517-9.

55 Katsanos AH, Filippatou A, Manios E, et al. Blood pressure reduction and secondary stroke prevention: a systematic review and metaregression analysis of randomized clinical trials. Hypertension 2017;69.

56 Rashid P, Leonardi-Bee J, Bath P. Blood pressure reduction and secondary prevention of stroke and other vascular events: a systematic review. Stroke 2003;34:2741-8.

57 Liu L, Wang Z, Gong L, et al. Blood pressure reduction for the secondary prevention of stroke: a Chinese trial and a systematic review of the literature. Hypertens Res 2009:32:1032-40.

58 Dimeo F, Pagonas N, Seibert F, et al. Aerobic exercise reduces blood pressure in resistant hypertension. Hypertension 2012:60:653-8.

59 Borghouts LB, Keizer HA. Exercise and insulin sensitivity: a review. Int I Sports Med 2000;21:1-12.

60 Lira FS, Yamashita AS, Uchida MC, et al. Low and moderate, rather than high intensity strength exercise induces benefit regarding plasma lipid profile. Diabetol Metab Syndr 2010;2:31

61 Rigotti NA, McKool KM, Shiffman S. Predictors of smoking cessation after coronary artery bypass graft surgery. Results of a randomized trial with 5-year follow-up. Ann Intern Med 1994;120:287-93.

62 Burn J, Dennis M, Bamford J, et al. Long-term risk of recurrent stroke after a first-ever stroke. The Oxfordshire community stroke project. Stroke 1994;25:333-7.

63 He FJ, Li J, Macgregor GA. Effect of longer term modest salt reduction on blood pressure: Cochrane systematic review and meta-analysis of randomised trials. BMJ 2013:346:f1325.

64 Kelley GA, Kelley KS. Effects of diet, aerobic exercise, or both on Non-HDL-C in adults: a meta-analysis of randomized controlled trials. Cholesterol 2012;2012:1-5.

65 Matthews CE, Hagströmer M, Pober DM, et al. Best practices for using physical activity monitors in population-based research. Med Sci Sports Exerc 2012;44:S68-S76.

66 British Association for Cardiovascular Prevention and Rehabilitation. BACPR standards and core components for cardiovascular disease prevention and rehabilitation 2012 2nd Edn, 2012. (accessed Oct 2017).

67 Jelinek MV, Thompson DR, Ski C, et al. 40 years of cardiac rehabilitation and secondary prevention in post-cardiac ischaemic patients. Are we still in the wilderness? Int J Cardiol 2015;179:153-9.

68 Menezes AR, Lavie CJ, Milani RV, et al. Cardiac Rehabilitation in the United States. Prog Cardiovasc Dis 2014;56:522-9.

69 Williamson W, Kluzek S, Roberts N, et al. Behavioural physical activity interventions in participants with lower-limb osteoarthritis: a systematic review with meta-analysis. BMJ Open 2015;5:e007642.

70 Heran B, Chen J, Ebrahim S, et al. Exercise-based cardiac rehabilitation for coronary heart disease. Cochrane Database of Systematic Reviews 2011;7:CD001800

71 Sagar VA, Davies EJ, Briscoe S, et al. Exercise-based rehabilitation for heart failure: systematic review and meta-analysis. Open Heart 2015;2:e000163. 